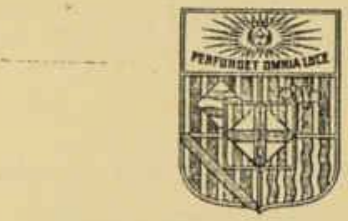

UNIVERSITAT DE BARCELONA

FACULTAT DE MATEMȦTIQUES

ANALYSIS OF SOME DEGENERATE QUADRUPLE COLLISIONS

by Carles Simó and Ernesto Lacomba

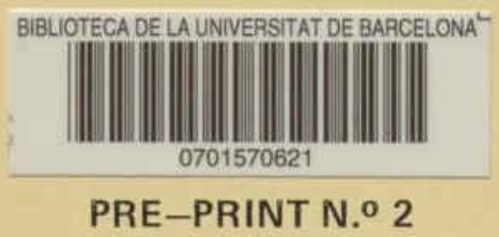





\section{Analysis of some degenerate quadruple collisions}

Carles Simó and Ernesto Lacomba**

- Facultat de Matematiques, Universitat de Barcelona, Spain.

* Departamento de Matemáticas, Universidad Autónoma Metropolitana, México.

Abstract.- We consider the trapezoidal problem of four bodies. This is a special problem where only three degrees of freedom are involved. The blow up method of McGehee can be used to deal with the quadruple collision. Two degenerate cases are studied in this paper: the rectangular and the collinear problems. They have only two degrees of freedom and the analysis of total collapse can be done in a way similar to the one used for the collinear and isosceles problems of three bodies. We fully analyze the flow on the total collision manifold, reducing the problem of finding heteroclinic connections to the study of a single ordinary differential equation. For the collinear case from which arises a one parameter family of equations the analysis for extreme values of the parameter is done and numerical computations fill up the gap for the intermediate values. Dynamical consequences for possible motions near total collision as well as for regularization are obtained.

51. Introduction. The trapezoidal problem of four bodies consists in the description of the motion of four particles of mases $m_{1}, m_{2}=$ $=m_{1}, m_{3}, m_{4}=m_{3}$ with initial coordinates $(a, b),(-a, b),(c, d)$ and ,$(-c, d)$, respectively and velocities such that the symmetry of coordi nates is keeped for all time. We can suppose that the center of masses remains at the origin, i.e., $m_{1} b+m_{3} d=0$. New variables $x=2 a, y=2 c, z=b-d$ can be introduced (see fig. 1). The motions near quadruple collision for that problem have been partially described

\section{fig. 1 here}

in [5]. In order to give a complete picture of the flow on the total collision manifold we restrict ourselves to two degenerate cases: the rectangular and the collinear. In the first the four masses

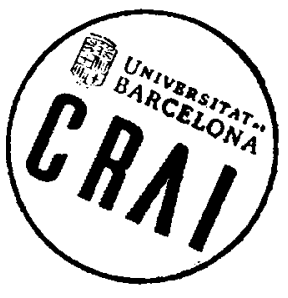


are equal and $a=c, b=d$. In the second one has $b=d=0$ but we sitill have one parameter: the mass ratio $a=m_{2} / m_{1}$. Then the total colli-sion mantfold is two dimensional (see [6] and [1]) and the invariant manifolds associated to the critical points are one dimensional. The study can be done on the same 1 ines as the one found in [6] and [7] for the collinear three body problem, or in [1],[8] and [2] for the isosceles problem. However the analysis of the behavior of the invariant manifolds is done using a single ordinary differential equation. A similar method was formulated in [4] and [3].

32. The rectangular case. First we set the masses equal to one for the bodies. We write down the Lagrangian

$$
L=1 / 2\left(\dot{x}^{2}+\dot{y}^{2}\right)-\frac{2}{x}-\frac{2}{y}-\frac{2}{\left(x^{2}+y^{2}\right)^{1 / 2}}
$$

and the corresponding Hamiltonian

$$
H=1 / 2\left(p_{x}^{2}+p_{y}^{2}\right)-\frac{2}{x}-\frac{2}{y}-\frac{2}{\left(x^{2}+y^{2}\right)^{1 / 2}}
$$

where the coordinates are described in fig. 2

fig. 2 here

The resulting Hamilton equations are

$$
\begin{array}{ll}
\dot{x}=p_{x}, & \dot{p}_{x}=-\frac{2}{x^{2}}-\frac{2 x}{r^{3}}, \\
\dot{y}=p_{y}, & \dot{p}_{y}=-\frac{3}{y^{2}}-\frac{2 y}{r^{3}},
\end{array}
$$

where $\quad c=\left(x^{2}+y^{2}\right)^{1 / 2}$.

Let us introduce the change of variables (see [6]):

$X=x \zeta^{-1}, \quad Y=y \zeta^{-1}, \quad P_{X}=p_{X}^{\zeta^{1 / 2}}, \quad P_{Y}=p_{Y} \zeta^{1 / 2}, \quad=\frac{d}{d \tau}=\zeta^{3 / 2} \frac{d}{d t}$

Then we have $x^{2}+Y^{2}=1$ and $\dot{\zeta}=c^{-1}\left(x p_{x}+y p_{y}\right)$. Introducing $V=x P_{x}+$ $+Y P_{Y}$ we get the blown up equations: 


$$
\begin{array}{ll}
X^{\prime}=P_{X}-X V & P_{X}^{\prime}=-\frac{2}{X^{2}}-2 X+Y_{2} V P_{X}, \\
Y^{\prime}=P_{Y}-Y V & P_{Y}^{\prime}=\frac{2}{Y^{2}}-2 Y+Y / 2 V P_{Y},
\end{array}
$$

On $\zeta=0$ (total collision manifold $C$ ) the equations are still regular and we shall use the description of the flow on $\mathrm{C}$ to get information about passage near total collision. As we know the change of variables is a diffeomorphism for $5>0$. The change has blown up the point $x=y=0$ to the manifold $c$. This has no physical meaning nor the fact that the new time $r$ on $C$ is obtained by and infinite slowing down of the physical time. However, the regularity of the equations on $C$ gives informations for small positive values of 5 and this has a clear physical importance.

On $C$ the equations of the epergy is $1 / 2\left(P_{X}^{2}+P_{Y}^{2}\right)-U=0$ where $U=\frac{2}{X}+\frac{2}{Y}+2$ and we get $V^{\prime}=U-\frac{V^{2}}{2}$.

The equilibrium points are $U_{c}=U_{\text {min }}=(X=Y=1 / \sqrt{2})=2+4 \sqrt{2}$ and $v_{c}= \pm \sqrt{8 \sqrt{2}+4}$.

We introduce a new change of coordinates: $X=\cos \theta, Y=\sin \theta$ and therefore

$$
X^{\prime}=-\sin \theta \cdot \theta^{\prime}, \quad \theta^{\prime}=-\frac{P_{X}-X V}{\sin \theta} .
$$

But $P^{2}=P_{X}^{2}+P_{Y}^{2}=2 U$ and $A r g \vec{P}=Y$ allow us to write $P \cos (\gamma-\theta)=$ $=\mathrm{V}$. Therefore

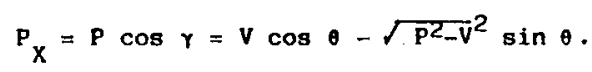

After substitution we have

$$
\theta^{\prime}= \pm \sqrt{2 V^{\prime}} \quad V^{\prime}=U(\theta)-\frac{V^{2}}{2}, \quad U(\theta)=\frac{2}{\cos \theta}+\frac{2}{\sin \theta}+2
$$

that: we integrate from $\theta=\pi / 4, \quad V=-\sqrt{8 \sqrt{2}+4}$ on to obtain the unstable manifold $w_{1}^{u}$ of the lower equilibrium point $A$ (see fig. 3 ). Now we have several possibilities for studying the equations of the manifold. We can obtain de/dV (see 13) or we can use the arc para meter $\sigma$ along $w_{1}^{u}$ as independent variable. The new equations are 


$$
\frac{d V}{d a}=\left(1+2 / V^{\cdot}\right)^{-1 / 2}, \quad \frac{d \theta}{d o}= \pm(1+v \cdot / 2)^{-1 / 2} \text {. }
$$

avoiding all the singularities. The change of sign in $\frac{d \theta}{d o}$ is produced when $\theta=0$ or $\pi / 2$.

\section{fig. 3 here}

53. Numerical computations and analytical estimations for the rectangular case. The last equations have been integrated starting at $A$ up to arrivirig to $V=0$ (point $B$ ). The values obtained are $\theta(B)=$ $=0.5877, \sigma(B)=4.459$. It is clear, using the symmetry with respect to $\theta=\pi / 4$ and $V=0$, that to have a connection between lower, $A$, and upper, $D$, equilibrium points requires $\theta$ (B) be a multiple of $\pi / 4$. The value 0.5877 is quite different from 0 and $\pi / 4$. However for people who deslikes results obtained through numerical integration we offer a proof of the fact that $w_{D}^{s} \neq w_{A}^{u}$ that involves only inequalities and a few evaluations of trigonometric and hyperbolic functions.

$$
\begin{aligned}
& \text { Dividing } \theta^{\prime} \text { by } V^{\prime} \text { we have } \\
& \qquad\left|\frac{d \theta}{d V}\right|=\sqrt{2 T V}=\sqrt{1 / \sec \theta+\operatorname{cosec} \theta+1-\frac{v^{2}}{4}} .
\end{aligned}
$$

we intend to show that starting at $B_{1}$ and going backwards we reach the curve $V=\sqrt{2 U(\theta)}$ to the right of the point $A$ and starting at $B_{2}$ we reach $\theta=\pi / 4$ above the point $A$ (see fig. 4)

fig. 4 here

- To prove the first part we show that this is true for a vectorfield F such that $\left|\frac{d \theta}{d V}\right| \leq F$ and for the second, that it is true for a $G$ such that $\left|\frac{d \theta}{d V}\right|>G$.

Let $k_{i}=\left[\theta_{i}^{m i n}(\sec \theta+\operatorname{cosec} \theta)\right.$. In this range of $\theta$ we take $F=1 / \sqrt{k_{1}+1-\frac{V^{2}}{4}}$. If we set $\frac{d \theta}{d V}=F$ one has $\Delta \theta=\int v_{i+1} d V / \sqrt{k_{i}+1-\frac{v^{2}}{4}}$. Letting $v=2 \sqrt{k_{1}+1} \sin$ a we obtain $\Delta \theta=2 \Delta \alpha$. Now ${ }^{\sqrt{ }}$ we split the range of in the following set of intervals (in degrees) $\left[0^{\circ}, 5^{\circ}\right]$, $\left[5^{\circ}, 15^{\circ}\right],\left[15^{\circ}, 30^{\circ}\right],\left[30^{\circ}, 60^{\circ}\right],\left[60^{\circ}, 75^{\circ}\right],\left[75^{\circ}, 85^{\circ}\right],\left[85^{\circ}, 95^{\circ}\right],\left[95^{\circ}, 105^{\circ}\right]$ $\left[105^{\circ}, 120^{\circ}\right],\left[120^{\circ}, 135^{\circ}\right]$. For angles greater then $90^{\circ}$ we take the 
symmetrical with respect to $90^{\circ}$. The points separing intervals are $\theta_{0}=0^{\circ}, \theta_{1}=5^{\circ}, \ldots,{ }_{9}{ }_{9}=120^{\circ}, Q_{0}=135^{\circ}$. At each one of such points we shall compute $v_{1}$. Note that for each $v_{1}$ we have two values of $a, a_{21-1}, a_{21}$, depending on the value of $k_{1}$ used, the one related to the left or right interval. Using symmetry and convexity $k_{0}=\sec 5^{\circ}+\operatorname{cosec} 5^{\circ}=k_{6}, \quad k_{1}=2 \sqrt{6}=k_{5}=k_{7}, k_{2}=2+2 / \sqrt{3}=$ $=k_{4}=k_{8}, k_{3}=2 \sqrt{2}=k_{9}$.

We set up te recurrence $\alpha_{2 i+1}=\alpha_{2 i}+\left(\theta_{i+1}-\theta_{i}\right) / 2$, $\sqrt{k_{i}+1} \sin a_{2 i+1}=\sqrt{k_{i+1}+1} \sin a_{2 i+2}, i=0 \div 8$, starting with $a_{0}=0$. A few computations of trigonometric functions give the values $a_{1}=\pi / 72, a_{3}=0.153246335, \alpha_{5}=0.313807297, a_{7}=0.589183175$, $a_{9}=0.693534686, a_{11}=0.653534211, \alpha_{13}=0.501227454, \alpha_{15}=.900181094$ $\alpha_{17}=1.335010689$ and then we obtain $\sin \alpha_{18}>1$ showing that under $F$ we reach the value $V=V_{c}$ to the right of point $A$.

Now we proceed to study the solutions of $\frac{d \theta}{d V}=G$ starting at $v=0, \theta=\pi / 4$. Consider the interval $[a, b] \subset[0, \pi / 4]$. Suppose that $v(a)<v(b)$. Then we take as $1 / G$ the function $\sqrt{\frac{d}{\theta}+\sec (a)+1-\frac{v(a)}{4}}^{2}$

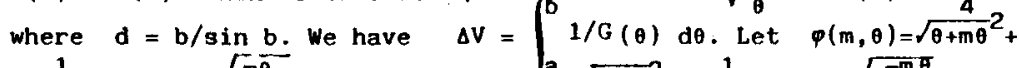
$+\frac{1}{\sqrt{m}} \operatorname{argtanh} \sqrt{\frac{m \theta}{1+m \theta}}$ if $m>0$ and $\sqrt{a+m \theta^{2}}+\frac{1}{\sqrt{-m}} \operatorname{arctg} \sqrt{\frac{-m \theta}{1+m \theta}}$ if $m \stackrel{\sqrt{m}}{<} 0$. Define $g=\sec (a)+1-\frac{V(a)^{2}}{4}$. Then $\Delta V=\sqrt{d}\left(\varphi\left(\frac{g}{d}, b\right)-\varphi\left(\frac{g}{d}, a\right)\right)$. When $\theta$ goes from $\pi / 4$ to $\pi / 2$ and again to $\pi / 4$ and $V$ decreases, the variation $\Delta V$ is equal to the variation obtained going from $\pi / 4$ to 0 and again to $\pi / 4$. Using the partition $[\pi / 6, \pi / 4],[\pi / 12, \pi / 6],[\pi / 36$, $\pi / 12],[0, \pi / 36]$ twice (the same partition used for $F$ ) we have

$$
\Delta V=\sum_{i=1}^{g} \sqrt{\mathrm{di}}\left(\varphi\left(\frac{g_{i}}{d i}, \theta_{i+1}\right)-\left(\frac{g_{1}}{d i}, \theta_{i}\right)\right),
$$

where in $g_{i}$ the value $v$ is taken as $\sum_{j=1}^{-1}$. The values $\theta_{i}$ are $\pi / 4 ; \pi / 6, \pi / 12$, etc. The evaluation of the $\mathrm{j}_{\mathbf{r}}^{\mathrm{r}} \mathbf{1}$ quired inverse trigonometric and hyperbolic functions gives $\Delta V=3.856090805<\sqrt{4+8 \sqrt{2}}=\left|v_{c}\right|$ proving that under $G$ we reach $\theta=\pi / 4$ on a point above $A$, as des 1 red. As a conclusion we have proved the following result. 
Theorem 2.1. The right part of the invariant unstable manifold of the lower equilibrium point $A$ reaches the value $v=0$ for $\theta \in(0, \pi / 4)$.

The conclusions about the remaining part of $w_{1}^{\mathrm{u}}$ can be obtai ned by symmetry. After a sequence of binary collisions (of course, couples of simultaneous double collisions) of types 1 and 2 (see fig. 5) slightly below or above the quadruple collision point $A$, the bodies escape as shown in fig. 5. A similar behavior is obtained for left hand side collisions.

\section{fig. 5 here}

54. The collinear case. Let $m_{1}=m_{2}=1, m_{3}=m_{4}=a$ be the masses of the four bodies and $x,-x, y / \sqrt{a},-y / \sqrt{\alpha}$ the coordinates (see fig. 6). We write down again the Lagrangian

$$
L=\dot{x}^{2}+\dot{y}^{2}-\frac{1}{2 x}-\frac{a^{5 / 2}}{2 y}-\frac{2 a^{3 / 2}}{y-x \sqrt{a}}-\frac{2 a^{3 / 2}}{y+x \sqrt{a}}
$$

and the corresponding Hamiltonian, setting, $p_{x}=2 \dot{x}, p_{y}=2 \dot{y}$,

$$
\begin{gathered}
H=\frac{p_{\dot{x}}^{2}}{y}+\frac{p_{y}^{2}}{y}-\frac{1}{2 x}-\frac{a^{5 / 2}}{2 y}-\frac{2 a^{3 / 2}}{y-x \sqrt{a}}-\frac{2 a^{3 / 2}}{y+x \sqrt{a}}=\frac{p_{x}^{2}}{y}+\frac{p_{y}^{2}}{y}-U(x, y) . \\
\text { fig. } 6 \text { here }
\end{gathered}
$$

Introducing $\zeta=\left(2 x^{2}+2 y^{2}\right)^{1 / 2}$ and the same change of coordinates of 52 we get again

$$
\begin{aligned}
& X^{\prime}=\frac{P_{X}}{2}-X V, \quad P_{X}^{\prime}=-\frac{1}{2 X^{2}}-\frac{2 a}{\left(\frac{Y}{\sqrt{a}}+X\right)^{2}}+\frac{2 a}{\left(\frac{Y}{\sqrt{a}}-X\right)^{2}}+1 / 2 V P_{X}, \\
& Y^{\prime}=\frac{P_{Y}}{2}-Y V, \quad P_{Y}^{\prime}=-\frac{a^{5 / 2}}{2 Y^{2}}-\frac{2 a^{1 / 2}}{\left(\frac{Y}{\sqrt{a}}-X\right)^{2}}-\frac{2 a^{1 / 2}}{\left(\frac{Y}{\sqrt{a}}+X\right)^{2}}+Y / 2 V P_{Y},
\end{aligned}
$$

where $V=X P_{X}+Y P_{Y}$ as before. We have again $V^{\prime}=U-1 / 2 V^{2} \quad$ on $\boldsymbol{\zeta}=0$. 
Introducing $X=\frac{1}{\sqrt{2}} \cos \theta, Y=\frac{1}{\sqrt{2}} \sin \theta$, the equation $\theta^{\prime}=$ $= \pm \sqrt{2 \mathrm{~V}}$, is obtained.

The equilibrium points are obtained in the following way: let $z=y / \sqrt{a}$. From $\frac{\ddot{x}}{x}=\frac{\ddot{z}}{z}$ and letting $z=\mu x$ we have

$$
\alpha=\frac{\mu^{3}\left(\mu^{2}-1\right)^{2}-8 \mu^{2}\left(\mu^{2}+1\right)}{17 \mu^{4}-2 \mu^{2}+1} .
$$

When a ranges from 0 to $\infty$ the parameter $\mu$ does between $\mu_{0}$ and $\infty$, where $\mu_{0}$ is the zero of $\mu\left(\mu^{2}-1\right)^{2}=8\left(\mu^{2}+1\right)$ (approximately $\left.\mu_{0}=2.396812289\right)$. The minimum value of $\theta$ is given by $\theta_{0}=\operatorname{arctg} \sqrt{a}$ and the critical one by ${ }_{c}=\operatorname{arctg}(\mu \sqrt{a})$.

In order to study the connection of the invariant manifolds starting at points $\left.\left(\theta_{c}, \pm \sqrt{2 U\left(\theta_{c}\right.}\right)\right)$ we introduce a new change of coor dinates (only useful for this purpose). Let $a=\frac{\pi / 2-\theta_{0}}{2}, b=\frac{\pi / 2+\theta_{0}^{-}}{2}$ and $\theta=b+a$ sin $\gamma$. Then we get

$$
\frac{d V}{d y}= \pm \frac{a}{\sqrt{2}} \sqrt{v^{\prime} \cos ^{2} r}
$$

and

$$
\begin{aligned}
v^{\prime} \cos ^{2} \gamma & =\left(\frac{a^{5 / 2}}{\sqrt{2} \sin \theta}+\frac{(2 \alpha)^{3 / 2} \cos \theta_{0}}{\sin \left(\theta+\theta_{0}\right)}-\frac{v^{2}}{2}\right) \cos ^{2} Y+ \\
& +\frac{1 / \sqrt{2} \cos ^{2} \gamma}{\sin (a(1-\sin \gamma))}+\frac{(2 \alpha)^{3 / 2} \cos \theta_{0} \cos ^{2} \gamma}{\sin (a(1+\sin \gamma))} .
\end{aligned}
$$

The term $\frac{\cos ^{2} \gamma}{\sin (a(1-\sin \gamma))}$ (and the one with the + sign in a - similar way) has an avoidable singularity. If $r=\frac{\pi}{2}+\varepsilon$, for instan ce, we merely write

$$
\frac{\cos ^{2} \gamma}{\sin (a(1-\sin \gamma))}=\frac{4 \cos ^{2} \varepsilon / 2}{\frac{\sin (2 a \psi)}{4}},
$$

where $=\sin ^{2} \varepsilon / 2$ and compute $\frac{\sin (2 a \psi)}{v}$ as $2 a-\frac{4}{3} a^{3} v^{2}+\frac{4}{15} a^{5} \psi^{4}-$ $-\frac{8}{315} a^{7}{ }^{6}+\ldots$ The computation must be started with $r=\gamma_{c}=$ $=\arcsin \left(\frac{\theta_{c}-b}{a}\right) \in\left(-\frac{\pi}{2}, \frac{\pi}{2}\right), v_{c}=-\sqrt{2 U\left(\theta_{c}\right)}$. In $\frac{d V}{d r}$ the + sign is used for the unstable manifold (right branch) and the - sign (with $\gamma$ decreasing) for the left branch. 
5. Numerical computations for the collinear case. Using: the equation numerically regularized as described in 4 we have computed the point $Y_{+}\left(\gamma_{-}\right)$where the right (left) branch of the unstable mani fold of the lower equilibrium point reaches the value $V=0$.

The independent parameter has been the parameter $\mu$. Table 1 shows some results. Figure 7 offers a rough representation of $\gamma_{ \pm}$as a function of a including the region of small values of a. The computations have been done using a $\mathrm{RK}$, routine of fourth order with step equal to 0.02 . Some errors can be introduced for this value of the step for large values of $a$.

fig. 7 here

In order to study the possible motions on the total collision manifold as a function of $a$ we need the connections between the equilibrium points. For $r_{+}=\frac{2 k-1}{2} \pi, k \in \mathbb{N}$ or $-\underline{r}=\frac{2 k+1}{2} \pi$, one of the branches of $w_{1}^{u}$ coincides $w i t h$ one of the branches of $w_{u}^{8}$. For $r_{+}-\underline{r}_{-}=2 k \pi_{1} k \in \mathbb{N}$, both branches meet due to the symmetry. Table 2 offers some values of $\alpha$ for which such connections are establi-shed.

6. Analytical study of the limiting cases. We study the behavior of $r_{+}, r_{-}$and, incidentally, $r_{c}, v_{c}$ for $a+0, \alpha+\infty$.

For $a=0$ we have $\theta_{0}=\theta c=0, v_{c}=-2^{1 / 4}, a=b=\pi / 4, r_{c}=-\pi / 2$. The $\therefore$ differential equation is $\frac{d v^{c}}{d \theta}=\frac{1}{2} \sqrt{\sqrt{2} \sec \theta-v^{2}}$, and scaling $v=2^{1 / 4} \vec{v}$ we get $\vec{v}^{2}+\left(\frac{d \bar{v}}{d \theta}\right)^{2} \cdot 4$ sec $\theta$ with $\bar{v}=-1$ for $\theta=0$.

Lemma. The solution of $\bar{v}^{2}+4\left(\frac{d \bar{v}}{d \theta}\right)^{2}=\sec \theta$ such that $\bar{v}(0)=-1$ reaches $\nabla=0$ for $\theta=n / 2$.

Proof: It is enough to check that the solution is given by $\bar{v}(\theta)=$ $=-\sqrt{\cos \theta}$.

Corollary. For $\alpha=0$ we have $\gamma_{+}=\pi / 2, \quad-\gamma_{-}=3 \pi / 2$. 


\begin{tabular}{|c|c|c|c|c|c|c|c|c|c|c|c|}
\hline$\mu$ & $a$ & $r_{c}$ & $v_{c}$ & $\gamma_{+}$ & ${ }^{r}$ & $\mu$ & $a$ & $Y_{c}$ & $\mathrm{v}_{\mathrm{c}}$ & $Y_{+}$ & ${ }^{Y}$ \\
\hline 2.39682 & .000008 & -1.4718 & -1.1892 & 1.5712 & -4.7120 & 3.3 & 1.24254 & .2775 & -5.0787 & 7.4640 & -6.5645 \\
\hline 2. 39685 & .000036 & -1.4244 & -1.1894 & 1.5729 & -4.7119 & 3.4 & 1.43195 & .3126 & -5.5993 & 8. 1957 & -6.8339 \\
\hline 2.3969 & .000086 & -1.3886 & $=1.1896$ & 1.5752 & -4.7118 & 3.5 & 1.63314 & .3442 & -6.1455 & 8.7929 & -7.1057 \\
\hline 2.397 & .000183 & -1.3501 & -1.1900 & 1.5789 & -4.7116 & 3.6 & 1.84650 & .3729 & -6.7191 & 9.2465 & -7.3841 \\
\hline 2.398 & .001155 & -1.2179 & -1.1940 & 1.6041 & -4.7107 & 3.7 & 2.07239 & .3992 & -7.3219 & 9.6424 & -7.6717 \\
\hline 2.400 & .003103 & -1.1153 & -1.2019 & 1.6416 & -4.7093 & 3.8 & 2.31119 & .4233 & -7.9556 & 10.016 & -7.9690 \\
\hline 2.402 & .005055 & -1.0534 & -1.2099 & 1.6733 & -4.7082 & 3.9 & 2.56326 & .4457 & -8.6220 & 10.385 & -8.2741 \\
\hline 2.406 & .008966 & -.9692 & -1.2259 & 1.7297 & -4.7064 & 4.0 & 2.82898 & .4666 & -9.3229 & 10.761 & -8.5845 \\
\hline 2.412 & .014856 & -.8833 & -1.2498 & 1.8054 & -4.7044 & 4.5 & 4.37492 & .5531 & -13.410 & 12.715 & -10.578 \\
\hline 2.420 & .022752 & -.8011 & -1.2815 & 1.8977 & -4.7026 & 5.0 & 6.31619 & .6195 & -18.648 & 15.986 & -13.400 \\
\hline 2.430 & .032692 & -.7237 & -1.3212 & 2.0055 & -4.7013 & 5.5 & 8.69752 & .6730 & -25.294 & 18.383 & -15.688 \\
\hline 2.438 & .040698 & -.6736 & -1.3523 & 2.0882 & -4.7011 & 6.0 & 11.5634 & .7176 & -33.628 & 22.057 & -19.281 \\
\hline 2.45 & .052802 & -.6106 & -1.4002 & 2. 2087 & -4.7018 & 6.5 & 14.9582 & .7556 & -43.946 & 24.966 & -22.134 \\
\hline 2.46 & .062974 & -.5660 & -1.4396 & 2.3070 & -4.7034 & 7.0 & 18.9261 & .7887 & -56.564 & 28.993 & -26.150 \\
\hline 2.47 & .073226 & -.5266 & -1.4789 & 2.4043 & -4.7057 & 7.5 & 23.5114 & .8178 & -71.813 & 32.716 & -30.459 \\
\hline 2.48 & .083555 & -.4913 & -1.5182 & 2.5008 & -4.7089 & 8.0 & 28.7583 & .8438 & -90.042 & 37.139 & -34.200 \\
\hline 2.49 & .093965 & -.4592 & -1.5575 & 2.5969 & -4.7128 & 8.5 & 34.7109 & .8672 & -111.616 & 41.624 & -38.698 \\
\hline 2.5 & .104456 & -.4297 & -1.5967 & 2.6930 & -4.7174 & 9.0 & 41.4134 & .8883 & -136.917 & 46.709 & -43.730 \\
\hline 2.6 & .213869 & -.2215 & -1.9896 & 3.6463 & -4.8028 & 9.5 & 48.9100 & .9077 & -166.343 & 50.925 & -47.973 \\
\hline 2.7 & .331810 & -.0904 & -2.3877 & 4.5299 & -4.9564 & 10.0 & 57.2446 & .9254 & -200.308 & 56.344 & -53.323 \\
\hline 2.8 & .458723 & .0051 & -2.7955 & 5.1956 & -5.1746 & 11.99 & 99.5948 & .9838 & -389.219 & 78.643 & -75.591 \\
\hline 2.9 & .595040 & .0800 & -3.2163 & 5.6878 & -5.4415 & 12.00 & 99.8476 & .9841 & -390.416 & 78.726 & -75.671 \\
\hline 3.0 & .741177 & .1414 & -3.6528 & 6.1072 & -5.7289 & 12.01 & 100.1008 & .9843 & -391.616 & 78.808 & -75.755 \\
\hline 3.1 & .897541 & .1933 & -4.1074 & 6.5080 & -6.0150 & 20.0 & 467.9 & 1.1196 & -2610.1 & 191.0 & -187.9 \\
\hline 3.2 & 1.06453 & .2381 & -4.5821 & 6.9345 & -6.2932 & 30.0 & 1584.4 & 1.2034 & -11917.9 & 366.9 & -363.8 \\
\hline
\end{tabular}




\begin{tabular}{|c|c|c|}
\hline k & $a_{k}$ & type \\
\hline 1 & .09297 & $-\gamma=3 \pi / 2$ \\
\hline 2 & .36153 & $\gamma^{-}=3 \pi / 2$ \\
\hline 3 & .90788 & $-\gamma=4 \pi$ \\
\hline 4 & 1.3452 & $5 \bar{\pi} / 2$ \\
\hline 5 & 2.2181 & $=5 * / 2$ \\
\hline 6 & 2.6362 & $\gamma-\gamma=6 \pi$ \\
\hline 7 & 2.9986 & $=7 \frac{t}{1} / 2$ \\
\hline 8 & 4.4984 & $=7 \pi / 2$ \\
\hline 9 & 4.8210 & $-\gamma=8 \pi$ \\
\hline 10 & 5.1229 & $9 \pi / 2$ \\
\hline 11 & 7.0515 & $-Y=9 \pi / 2$ \\
\hline 12 & 7.3237 & $r^{-}-r_{-}=10 \pi$ \\
\hline 13 & 7.5859 & $\gamma^{+}=1 \overline{1}_{\pi} / 2$ \\
\hline 14 & 9.8469 & $-\gamma^{+}=11 \pi / 2$ \\
\hline 15 & 10.0878 & $r_{+}^{-} r_{-}=12 \pi$ \\
\hline
\end{tabular}

\begin{tabular}{|c|c|c|}
\hline k & $a_{k}$ & $\therefore$ type \\
\hline 16 & 10.3230 & $\bar{\gamma}=13 \pi / 2$ \\
\hline 17 & 12.8688 & $-\gamma^{+}=13 \pi / 2$ \\
\hline 18 & 13.0880 & $r=14 \pi$ \\
\hline 19 & 13.3035 & $5 \pi / 2$ \\
\hline 20 & 16.1072 & $5 \pi / 2$ \\
\hline 21 & 16.3105 & $=16 n$ \\
\hline 22 & 16.5115 & $1 \overline{7}: / 2$ \\
\hline 23 & 19.5572 & $=17 \pi / 2$ \\
\hline 24 & 19.7484 & $\gamma=18 n$ \\
\hline 25 & 19.9379 & \\
\hline 26 & 23.215 & $-\gamma=19 \pi / 2$ \\
\hline 27 & 23.397 & $\gamma^{-}-\gamma=20 \pi$ \\
\hline 28 & 23.578 & $\gamma^{+}=2 \overline{1} \pi / 2$ \\
\hline 29 & 27.080 & $-r^{+}=21 \pi / 2$ \\
\hline 30 & 27.254 & $r_{+}-r_{-}=22 \pi$ \\
\hline 31 & 27.427 & $r_{+}=23 \pi / 2$ \\
\hline
\end{tabular}

Table 2 
Now we study what happens for $a>0$ sufficiently small. First of all we have, approxigatel $y_{3} /{ }^{\theta}{ }_{0}=\sqrt{a}{ }_{*} \theta_{c}=\mu_{0} \sqrt{a}$. Therefore $U\left(\theta_{c}\right)=$ $=\frac{1}{\sqrt{2}}+a\left(\frac{1}{\sqrt{2}} \frac{\mu_{0}^{2}}{2}+\frac{2^{3 / 2}}{\mu_{0}+1}+\frac{2^{3 / 2}}{\mu_{0}-1}\right) \alpha+o\left(a^{2}\right)^{c}$ and, $v_{c}=-\sqrt{2}-\sqrt{2}\left(\frac{\mu a^{c}}{4}+\frac{2}{\mu_{0}+1}+\right.$ $\left.\frac{2}{\mu_{0}-1}\right) \alpha+O\left(\alpha^{2}\right)$. In order to check the behavior observed in 35 we have to prove two things: $r_{+}>\pi / 2,-r_{-}<3 \pi / 2$.

We start at $P(V=0, \theta=x / 2)$ and follow the differential equa tion $\frac{d V}{d \theta}=\sqrt{\frac{U(\theta)}{2}-\frac{V^{2}}{4}}$ backwards.

Writting down $v=-2^{1 / 4} \sqrt{\cos \theta}+w, w(\pi / 2)=0$ and retalning first order terms we get:

$$
\frac{d w}{d \theta}=\frac{w}{2^{5 / 4}} \frac{\cos \theta}{\sin \theta}+\frac{2 a^{3 / 2} \cos ^{1 / 2} \theta}{\sin \theta}\left(\frac{1}{\sin \left(\theta+\theta_{0}\right)}+\frac{1}{\sin \left(\theta-\theta_{0}\right)}\right) .
$$

The solution of the homogeneous equation is $w=C(\sin \theta)^{-1 / 2^{5 / 4}}$ and the method of variation of the constants gives us

$$
\frac{d c}{d \theta}=\frac{2 a^{3 / 2} \cos ^{1 / 2} \theta}{(\sin \theta)}\left(1-1 / 2^{5 / 4}\right)\left(\frac{1}{\sin \left(\theta+\theta_{0}\right)}+\frac{1}{\sin \left(\theta-\theta_{0}\right)}\right) .
$$

Therefore $w\left(\theta_{c}\right)=-\left(\mu_{0} \sqrt{a}\right)^{-1 / 2^{5 / 4}} \Delta c$, where $\Delta_{c} c=\int_{\theta_{c}}^{\pi / 2} \frac{d c}{d \theta_{z}}$. The value
$\Delta c$ can be estimated in the following way $\int_{\theta}^{\pi / 2}=\int_{\theta c}^{\pi / 2} z$,
where $z$ is a small but finite quantity and so

$$
\int_{z}^{\pi / 2}=O\left(a^{3 / 2}\right) \text {. }
$$

It remains to compute the main contribution $\int_{1}^{2 \sin \theta} \int_{\theta_{c}}$. We bound $\cos ^{1 / 2} \theta$ by 1, put $\frac{1}{\sin \left(\theta+\theta_{0}\right)}+\frac{1}{\sin \left(\theta-\theta_{0}\right)}<\frac{2 \sin \theta \sqrt{\theta_{c}}}{\sin \left(\theta+\theta_{0}\right) \sin \left(\theta-\theta_{0}\right)}$ and approximate the sinus by the angles. We get

$$
\begin{aligned}
& \Delta c=\int_{\theta c}^{z} \frac{4 a^{3 / 2} \theta^{1 / 2^{5 / 4}}}{\theta^{2}-a}<4 a^{3 / 2} \frac{\mu_{0}^{2}}{\mu_{0}^{2}-1} \int_{\theta c}^{z} \theta^{-2+1 / 2^{5 / 4}}= \\
& =\frac{4 a^{3 / 2} \mu_{0}^{2}}{\left(1-1 / 2^{5 / 4}\right)\left(\mu_{0}^{2}-1\right)}\left(\mu_{0} \sqrt{\alpha}\right)^{-1+1 / 2^{5 / 4}} .
\end{aligned}
$$


Then $w\left(\theta_{c}\right)=-\frac{4 a \mu_{0}}{\left(1-1 / 2^{5 / 4}\right)\left(\mu_{0}^{2}-1\right)}$. As $\frac{{ }^{4} \mu_{0}}{\left(1-1 / 2^{5 / 4}\right)\left(\mu_{0}^{2}-1\right)}<$ $<2^{1 / 4}\left(\frac{\mu_{0}^{2}}{4}+\frac{2}{\mu_{0}+1}+\frac{2}{\mu_{0}^{-1}}\right)$ the point $Q$ (fig. 8) is above the equi librium point ${ }^{\circ}\left(\theta_{c}, \theta_{c}\right)$, showing that $r_{+}>\pi / 2$.

Now let us look for the point $R$ (see fig. 8). The first order terms for $\gamma_{c}$ give us $\gamma_{c}=-\frac{\pi}{2}+\sqrt{\frac{8\left(\mu_{0}-1\right)}{\pi}} \alpha^{1 / 4}$. On the other side the main term in $\frac{d V}{d \gamma}$ is $-\sqrt{\pi / 4 \sqrt{2}}$ near the left hand side coll1 sion. Therefore the value of $\Delta V$ from the point $\left(\theta_{c}, V_{c}\right)$ to $R$ is $2 \sqrt{\pi / 4 \sqrt{2}}\left(\pi / 2+\gamma_{c}\right)=\sqrt{8\left(\mu_{0}^{-1)} / \sqrt{2}\right.} a^{1 / 4}$, showing that $-\gamma_{-}<3 \pi / 2$. We have proved the following result.

Proposition 6.1. For a small enough $\gamma_{+}>\pi / 2,-\gamma_{-}<3 \pi / 2$.

For a large we have $\theta_{0}=\frac{\pi}{2}-\frac{1}{\sqrt{\alpha}}, \theta_{c}=\frac{\pi}{2}-\frac{1}{\sqrt{a} \frac{1}{17 g}}$. Introducing $\nabla=$ $=v / a^{5 / 4}$ and retaining the dominant term in the differential equa-tion we get $\cdot v_{c}=-a \sqrt{2 a}, \frac{d \vec{v}}{d y}=\frac{a}{\sqrt{2}} \sqrt{1-\frac{\vec{v}^{2}}{\sqrt{2}}}|\cos \gamma|$ where $a=\frac{1}{2 \sqrt{a}}$.

$$
\int_{-\sqrt{2}}^{0} \frac{d \bar{V}}{\sqrt{1-\bar{v}^{2} / 2}}=\frac{a}{\sqrt{2}} T \frac{2}{\pi}
$$

where $T$ is the $Y$ interval and $\frac{2}{\pi}$ is the average value of $|\cos \gamma|$. we get immediately $T=2^{-1 / 4} \pi^{2} a^{1 / 2}$. We state the result, showing good agrement with table 1 .

Proposition 6.2. For a sufficiently large $\gamma_{+}=\pi^{2} 2^{-1 / 4} \alpha^{1 / 2}$ and $r_{+}+\gamma_{-} \rightarrow \pi$.

Corollary 6.3. There are infinite values for which the left hand - branch of $w_{1}^{u}$ coincides with the left hand branch of $w_{u}^{s}$ and for which the right hand one coincides with the right hand one and for which $w_{1}^{u} \equiv w_{u}^{s}$. In the last case the left hand branch of $w_{1}^{u}$ coincides with the right hand one of $w_{u}^{s}$ and viceversa.

The first one values for which those coincidences are obtained were given in table 2 . 
57. Some dynamical consequences. Let $a_{1}$ be the unique value $a>0$ such that $-\gamma_{-}\left(\alpha_{1}\right)=3 \pi / 2, \alpha_{2}$ such that $\gamma_{+}\left(\alpha_{2}\right)=3 \pi / 2, \alpha_{3}$ such that $r_{+}\left(a_{3}\right)-r_{-}\left(a_{3}\right)=4$, etc. Figure 9 shows a qualitative picture of the invariant manifolds of the lower equilibrium point for a initial range of values of a containing those values $\left(0<a_{1}<a_{2}<a_{3}\right)$.

Fig. 9 here

The consequences with respect to orbits passing near quadruple collision are now obtained easily in the same way as they where obtatned for the rectangular case (see orbits type 1,2 in fig. 3). We recall that other necessary conditions for regularization can be obtained (for the good values of $\alpha$, 1.e., such that $w_{1}^{u} \equiv w_{u}^{5}$ )in the way introduced in [7]. Sufficient conditions will be given in a forthcoming paper [9].

From fig. 9 the way of escaping after approaching a quadruple collision and the number of collisions taking place between central bodies or simulateous double collisions between external bodies can be predicted.

Pictures similar to fig. 9 can be given for the full range of values of $a$. (Note that according to table 2 there is $a_{4}$ similar to $\alpha_{2} \quad \alpha_{5}$ similar to $\alpha_{1}$ and $a_{k}$ similar to $q_{k-3}$ for all $k>6$ ).

a. Acknowledgements. This work was initiated when both authors were visiting the Université de Dijon (France). The first author has been - partially supported by an Ajut a l'Investigació of the Universitat de Barcelona. The second author has been partially supported by the Grant PCCBNAL 790178 of the CONACYT (México). The computations were done at the Universitat Autonnoma de Barcelona and at the IMPA (Brasi1): 


\section{References}

[1] Devaney, R.: In Ergodic Theory and Dynamical Systems I, Ed. A. Katok, 211, Birkhauser, Basel 1981.

[2] Devaney, R.: These Proceedings.

[3] Irigoyen,M.: Celestial Mechanics, 9, 491.

[4] Irigoyen, M. and Nahon, F.: Astron. Astrophys. 17, 286.

[5] Lacomba, E.: To appear in Colloque Bifurcations, Théorie Ergodique et Applications, Dijon 1981 .

[6] McGehee, R.: Inventiones Math. 27, 191.

[7] Simó, C.: Celestial Mechanics, 21, 25.

[8] Simó, C.: In Classical Mechanics and Dynamical Systems, Marcel Dekker, New York, 1981.

[9] Simó, C.: Necessary and sufficient conditions for the geometrical regularization of blown up singularities, to appear, 1982. 

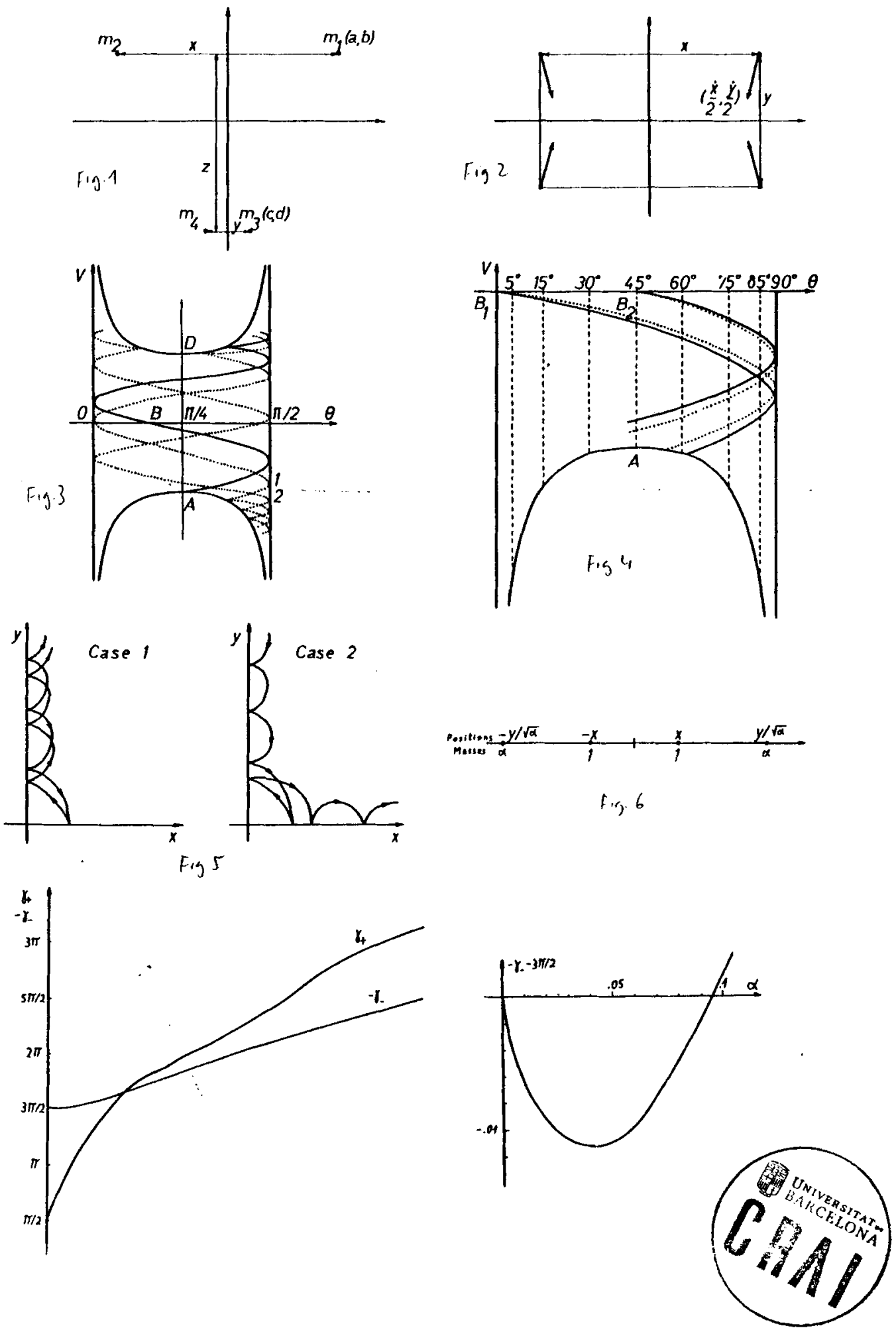

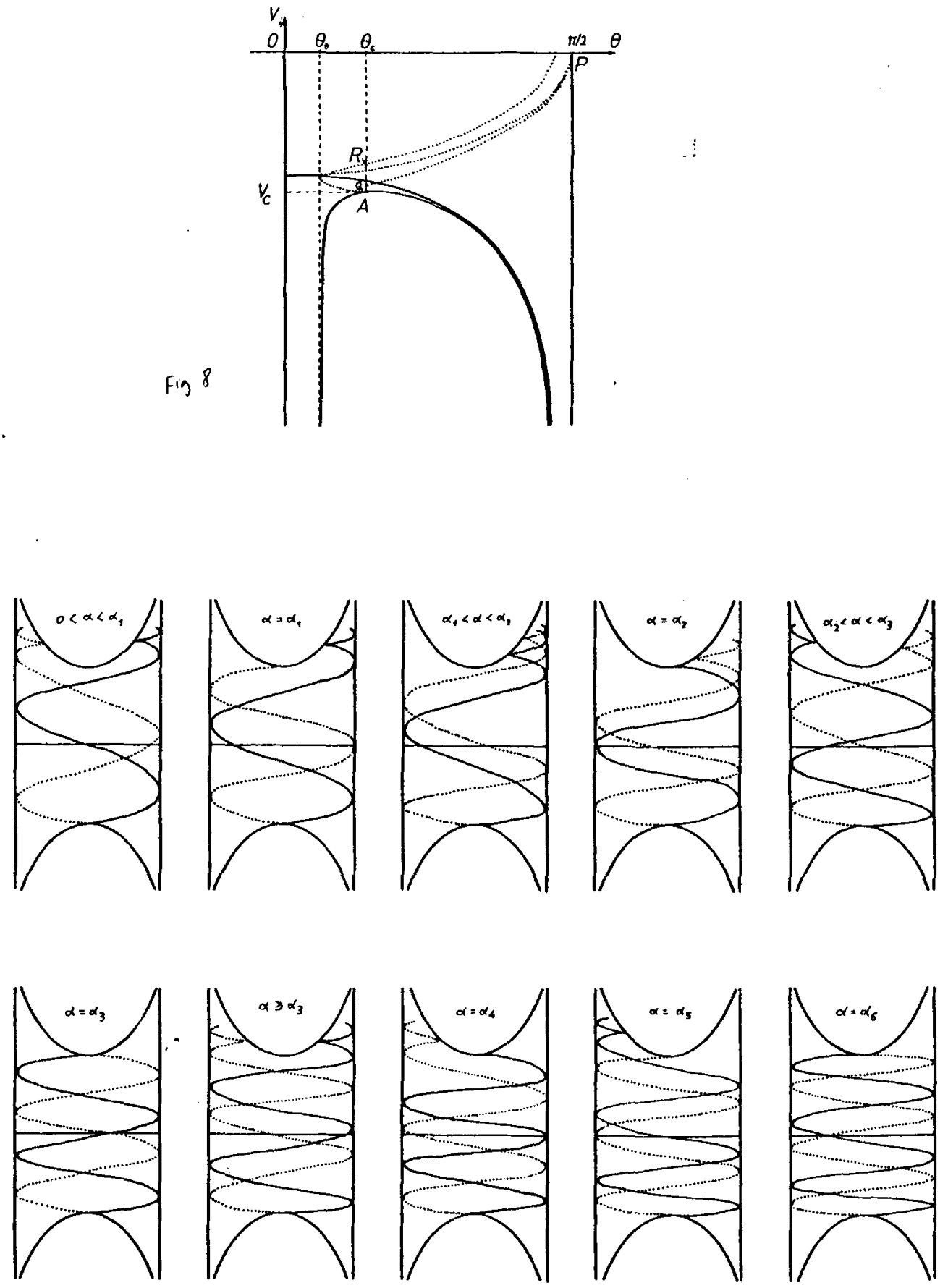

$\operatorname{lig} 9$ 

publicacions edicions

universitat

de barcelona

Diposit Legal B.: 4.223-1982

BARCELONA-1982 